Review Article

\title{
Ovum Pick Up and In Vitro Maturation of Jennies Oocytes Toward the Setting Up of Efficient In Vitro Fertilization and In Vitro Embryos Culture Procedures in Donkey (Equus asinus)
}

\author{
Stefan Deleuze a, *, Cécile Douet b, c, d, e, Isabelle Couty b, c, d, e, Carla Moros-Nicolás b, c, d, e , \\ Philippe Barrière ${ }^{\mathrm{f}}$, Thierry Blard ${ }^{\mathrm{f}}$, Fabrice Reigner ${ }^{\mathrm{f}}$, Michèle Magistrini ${ }^{\mathrm{b}, \mathrm{c}, \mathrm{d}, \mathrm{e} \text {, }}$ \\ Ghylène Goudet ${ }^{\mathrm{b}, \mathrm{c}, \mathrm{d}, \mathrm{e}}$ \\ a Faculté de Médecine Vétérinaire, Département des Sciences Cliniques-Clinique Equine, Université de Liège, Liège, Belgium \\ b INRA, UMR 85, Physiologie de la Reproduction et des Comportements, Nouzilly, France

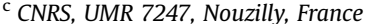 \\ d IFCE, Nouzilly, France \\ e Université François Rabelais, Tours, France \\ ${ }^{\mathrm{f}}$ INRA, UE1297 Unité Expérimentale de Physiologie Animale de l'Orfrasière, Nouzilly, France
}

\section{A R T I C L E I N F O}

\section{Article history:}

Received 16 October 2017

Received in revised form

3 March 2018

Accepted 4 March 2018

Available online 14 March 2018

\section{Keywords:}

Oocyte

Spermatozoa

Donkey

Ovum pick up

IVM

IVF

\begin{abstract}
A B S T R A C T
Most wild and domestic donkey breeds are currently endangered or threatened. Their preservation includes the creation of a Genome Resource Bank. Embryo cryopreservation allows preservation of genetics from both male and female and is the fastest method to restore a breed. Embryo production in vivo is limited in equids. We recently established a technique of ovum pick up (OPU) in the donkey. Conditions of in vitro maturation (IVM), in vitro fertilization (IVF), and in vitro culture of zygotes have been evaluated. Equine abattoir-derived oocytes were used as controls. Donkey cumulus-oocyte complexes (COCs) collected by OPU were matured in vitro in TCM199 with fetal calf serum and epidermal growth factor for $24,30,34$, or 38 hours. Forty-four percent were in metaphase 2 after 34 hours. In our conditions, IVM of donkey oocytes was slower than that of equine oocytes and the optimal duration for donkey oocytes IVM may be 34 hours. Oocytes we co-incubated with frozen-thawed donkey semen treated with procaine for 18 hours and cultured for 30 hours in a Dulbecco Modified Eagle Medium-F12based medium. Only $15 \%$ of jennies oocytes contained 2 pronuclei after co-incubation, and none of them developed further after 48 hours after IVF. Treatment of donkey sperm with procaine may not be efficient for IVF. Some parthenogenetic activation occurred. In conclusion, we confirm that our conditions for OPU in jennies yielded high recovery rates that improved with operator experience. Maturation rates of $44 \%$ can be achieved using the IVM medium routinely used for equine oocytes in our lab. Further studies are in progress to establish efficient conditions for IVF and development of donkey zygotes.
\end{abstract}

๑) 2018 Elsevier Inc. All rights reserved.

\footnotetext{
Animal welfare/ethical statement: All procedures on animals were conducted in accordance with the Guidelines for the Care and Use of Laboratory Animals issued by the French Ministry of Agriculture and with the approval of the Ethical Review Committee (Comité d'Ethique en Expérimentation Animale Val de Loire) under number 02701.01 .

Conflict of interest statement: The authors declare no conflicts of interest.

* Corresponding author at: Stefan Deleuze, Faculté de Médecine Vétérinaire, Département des Sciences Cliniques-Clinique Equine, Université de Liège, Liège, Belgium.

E-mail address: s.deleuze@ulg.ac.be (S. Deleuze).
}

\section{Introduction}

Several domestic breeds of donkeys such as the Asinina de Miranda donkey in Portugal [1], the Martina Franca donkey in Italy $[2,3]$, the Zamorano-Leonés donkey in Spain [4], and the American Mammoth Jack donkey in the United States [5] are vulnerable to extinction. In France, some breeds such as the "Grand Noir du Berry" donkey or the "Normand" donkey are even nearly extinct with fewer than 100 registered active breeding jennies (communication from the Institut Français du Cheval et de l'Equitation: http://statscheval.haras-nationaux.fr/core/zone_menus.php?zone= 229\& $\mathrm{r}=1316)$. 
Options to preserve endangered donkey breeds include the creation of a Genome Resource Bank. This requires cryopreservation of semen, oocytes, and/or embryos. Embryo cryopreservation allows preservation of genetics from both the male and the female and is the fastest method to restore a breed [6].

In vivo donkey embryo production after natural mating or artificial insemination has been reported previously with an embryo recovery rate per cycle from $50 \%$ to $76 \%[7,8]$. However, in vivo collection in equids is limited by the lack of an effective treatment for induction of multiple ovulations [5,9]. To our knowledge, no efficient superovulation protocol has been reported in the donkey. This seriously limits the number of embryos available for cryopreservation in that species. The size of the embryos at collection is also a limiting factor, as smaller embryos are more tolerant to the damage induced by cryopreservation [10]. In vitro produced embryos would be ideal for cryobanking as their size at the time of cryopreservation could be controlled. The first step toward in vitro embryo production (IVP) of donkeys is the availability of more oocytes. Retrieving several immature oocytes from a donor by ultrasound-guided ovum pick up (OPU) is widely reported in horses [10-20] and has been recently described in the donkey [21].

These immature oocytes collected by OPU require further in vitro maturation (IVM). Two reports about IVM of abattoirderived donkey oocytes have been published $[22,23]$. The optimal duration of in vitro culture has not been evaluated in these reports. The third step is in vitro fertilization (IVF) of oocytes. To date, despite a significant amount of research in that area, no efficient conventional IVF technique has been established in the equine [24-30]. In 2009, a 60\% rate of equine IVF was reported after treatment of fresh spermatozoa with procaine [31]. However, procaine was reported to induce cytokinesis in horse oocytes [32]. Part of the present article has been previously published in a report where similar procaine-based hyperactivation treatments were used for conventional IVF in the donkey [21]. The last step of IVP is the in vitro culture of the embryo to a developmental stage that allows cryopreservation. To date, no report on the optimization of culture media and conditions for donkey embryos have been published. Assisted reproduction in the donkey is still very poorly documented, and specifics of the OPU, oocytes IVM, IVF, and IVP still need to be investigated in the donkey.

This article presents the collection rates of OPU sessions performed on jennies and some data on our first attempts on IVM and IVF of donkey oocytes.

\section{Materials and Methods}

All chemicals were purchased from Sigma-Aldrich (Saint Quentin Fallavier, France) unless otherwise indicated.

\subsection{Collection of Donkey Immature Oocytes}

Donkey immature cumulus-oocyte complexes (COCs) were collected during three consecutive breeding seasons by transvaginal ultrasound-guided aspiration on the nine adult cyclic jennies from our experimental stud farm. Ovarian activity was assessed by routine rectal ultrasound scanning to choose jennies with several growing follicles from 5 to $25 \mathrm{~mm}$ and no corpus luteum. Follicles were punctured by transvaginal ultrasoundguided aspiration with a double-lumen needle (length $700 \mathrm{~mm}$, outer diameter $2.3 \mathrm{~mm}$, internal diameter $1.35 \mathrm{~mm}$, Casmed, Cheam, Surrey, England) and a sectorial probe (Aloka SSD900) as previously described [13]. After follicular fluid aspiration, the follicle was flushed with phosphate buffered saline (PBS, Dulbecco A, Oxoid, Basingstoke, Hampshire, England) and heparin (Choay, Sanofi Aventis $5000 \mathrm{IU} / \mathrm{mL}$ ) at $38^{\circ} \mathrm{C}$. All aspirated fluids were examined for oocyte recovery, and oocytes denuded of cumulus or degenerated oocytes showing shrunken, dense or fragmented cytoplasm were discarded. During the collection procedure, jennies were injected detomidine (Medesedan, $0.25 \mathrm{~mL} /$ animal i.v., $10 \mathrm{mg} /$ $\mathrm{mL}$ detomidine, Centravet; Plancoet, France) and butorphanol (Dolorex, $0.6 \mathrm{~mL} /$ animal i.v., $10 \mathrm{mg} / \mathrm{mL}$ butorphanol tartrate and $0.1 \mathrm{mg} / \mathrm{mL}$ benzethonium chloride, Centravet) for sedation and analgesia and dipyrone and butylscopolamine (Estocelan, $30 \mathrm{~mL} /$ animal i.v., $4 \mathrm{mg} / \mathrm{mL}$ butylscopolamine, Centravet) for analgesia and antispasmodia. After puncture, jennies received a preventive antibiotic injection (Depocilline, $20 \mathrm{~mL} /$ animal i.m., benzylpenicillin $170.41 \mathrm{mg} / \mathrm{mL}$, Intervet, Beaucouze, France) in 2015 and 2016 only. Ovum pick up sessions were conducted between June and early September.

\subsection{Collection of Horse Immature Oocytes}

Equine immature COCs were collected during the breeding season from slaughtered mares in commercial abattoirs. Ovaries from females of unknown reproductive history and breed were obtained at local commercial abattoirs immediately after females were killed. They were transported to the laboratory within 2 hours in $0.9 \%(\mathrm{w} / \mathrm{v}) \mathrm{NaCl}$ at $32^{\circ} \mathrm{C}-38^{\circ} \mathrm{C}$. Cumulus-oocyte complexes were collected using the aspiration procedure as previously described by Goudet et al [33]. Briefly, the tunica albuginea was removed, and all follicles larger than $5 \mathrm{~mm}$ were aspirated with an 18 gauge needle at $100 \mathrm{~mm} \mathrm{Hg}$ of vacuum pressure, then the ovaries were cut into thick sections with a scalpel blade to find other follicles within the ovarian stroma. Follicular fluids were examined under a stereomicroscope for COCs recovery. Oocytes denuded of cumulus and degenerated oocytes showing shrunken, dense, or fragmented cytoplasm were discarded. To avoid contaminations, COCs were washed in a medium containing antibiotics (Medium 199 with Earle's salts, 25 mM HEPES and $\mathrm{NaHCO}_{3}$ supplemented with $20 \%$ (v/ v) fetal calf serum (FCS) and $25 \mu \mathrm{g} / \mathrm{mL}$ gentamycin). Equine abattoir-derived oocytes were used as control for IVM conditions.

\subsection{In Vitro Maturation of Horse and Donkey Immature Oocytes}

Donkey and horse COCs were washed in maturation medium: Medium 199 with Earle's salts supplemented with 20\% (v/v) FCS and $50 \mathrm{ng} / \mathrm{mL}$ epidermal growth factor (EGF) [33]. They were cultured in groups of $10-30$ in $500 \mu \mathrm{L}$ of maturation medium in an atmosphere of $5 \% \mathrm{CO}_{2}$ in air at $38.5^{\circ} \mathrm{C}$ in $100 \%$ humidity. Jennies COCs were cultured for $24,30,34$, or 38 hours. Equine COCs were cultured for 24 or 30 hours.

\subsection{Donkey Semen Collection, Freezing, and Evaluation}

The study included 2 mature donkeys (Grand Noir du Berry) of proven fertility, aged 7 and 14 years. The donkeys were housed at INRA in Nouzilly, France. Semen was collected on a regular basis (three times a week) with a closed artificial vagina (INRA model). After collection, raw semen was filtered through gauze to remove the gel fraction of the ejaculate and immediately frozen in INRA Freeze extender according to the procedure described by Pillet et al [34]. The straws were stored in liquid nitrogen until used.

Donkey semen evaluation was performed as follows. Three straws per ejaculate and per donkey were thawed in a water bath for 30 seconds at $37^{\circ} \mathrm{C}$ and diluted in INRA 96 extender at $20 \times 10^{6}$ spermatozoa $/ \mathrm{mL}$. The diluted sperm cells were incubated at $37^{\circ} \mathrm{C}$ for 10 minutes, and motility parameters were evaluated using a computer-assisted analysis (IVOS, version 10, Hamilton Thorne; Beverly, MA). The parameters analyzed were average path velocity (VAP, $\mu \mathrm{m} \mathrm{s}^{-1}$ ), percent progressive sperm cells (PROG, VAP higher 
than $40 \mu \mathrm{m} \mathrm{s}^{-1}$, and straightness of track higher than $80 \%$ ), and percent rapid sperm cells (RAPID, VAP higher than $40 \mu \mathrm{m} \mathrm{s}^{-1}$ ). Analyses were performed in duplicate $(2 \times 3 \mu \mathrm{L}$ sampling/straw $)$ on three fields analyzed (i.e., six observations/straw and 18 observations/donkey/ejaculate). The motility parameters of the two ejaculates used for IVF (one per donkey) were as follows: VAP $121 \pm$ $0.7 \mu \mathrm{m} /$ second and $96 \pm 4.3 \mu \mathrm{m} / \mathrm{second}$; RAPID $67 \pm 3.4$ and $69 \pm$ $1.8 \%$; PROG $51 \pm 4.4 \%$ and $47 \pm 2.4 \%$.

\subsection{In Vitro Fertilization Procedure}

\subsubsection{Preincubation of Oocytes With Oviductal Fluid}

After 27 hours IVM, COCs were partially denuded by flushing and washed in modified Whitten's Medium (MW; $100 \mathrm{mM} \mathrm{NaCl}$, $4.7 \mathrm{mM} \mathrm{KCl}, 1.2 \mathrm{mM} \mathrm{MgCl}$, $22 \mathrm{mM}$ HEPES, $4.8 \mathrm{mM}$ lactic acid hemicalcium salt, $1 \mathrm{mM}$ pyruvic acid) supplemented with $5.5 \mathrm{mM}$ glucose (anhydrous), $25 \mathrm{mM} \mathrm{NaHCO}$, and $7 \mathrm{mg} / \mathrm{mL} \mathrm{BSA}, \mathrm{pH} 7.25$ [31].

COCs were then preincubated in droplets of $30 \mu \mathrm{L}$ of porcine oviductal fluid for 30 minutes in an atmosphere of $5 \% \mathrm{CO}_{2}$ in air at $38.5^{\circ} \mathrm{C}$ in $100 \%$ humidity, as previously described [35]. For porcine oviductal fluid collection, genital tracts from gilts with both ovaries containing several follicles larger than $5 \mathrm{~mm}$ were used. They were obtained at a commercial abattoir and transported to the laboratory at room temperature. The oviducts were dissected free from surrounding tissues. The oviductal content from the ampulla was expelled by gentle squeezing using a sterile microscope slide and collected by introducing the tip of a pipette into the ampulla and aspirating while making a manual ascendant pressure from the isthmus to the ampulla [36]. After centrifugation at $10,000 \times \mathrm{g}$ for 15 minutes, the supernatant containing secreted and intracellular components was immediately stored at $-20^{\circ} \mathrm{C}$ until used as "oviductal fluid".

\subsubsection{Preparation of Donkey Jack Sperm and In Vitro Fertilization}

Frozen donkey semen $\left(100 \times 10^{6} / \mathrm{mL}\right)$ from a single ejaculate of two jacks (two straws per jack) was thawed at $37^{\circ} \mathrm{C}$ for 30 seconds and pooled. Then, $2 \mathrm{~mL}$ of semen was diluted in $2 \mathrm{~mL}$ of prewarmed MW supplemented with $5.5 \mathrm{mM}$ glucose (anhydrous), pH 7.25 [31]. Diluted sperm was transported to the laboratory within a few minutes at $37^{\circ} \mathrm{C}$ and centrifuged in $15 \mathrm{~mL}$ conical tubes at $100 \times \mathrm{g}$ for 1 minute at $37^{\circ} \mathrm{C}$ to remove particulate matter and dead sperm. The supernatant was then transferred to a $14 \mathrm{~mL}$ round bottom tube and centrifuged at $600 \times g$ for 5 minutes at $37^{\circ} \mathrm{C}$. The pellet was resuspended in $1.5 \mathrm{~mL}$ of prewarmed MW supplemented with glucose, and the concentration was determined by counting on a Thoma chamber under a microscope (Olympus, IMT-2, Paris, France). Spermatozoa were then diluted at $10 \times 10^{6} / \mathrm{mL}$ in prewarmed MW supplemented with $5.5 \mathrm{mM}$ glucose, $25 \mathrm{mM} \mathrm{NaHCO}_{3}$, and $7 \mathrm{mg} / \mathrm{mL}$ BSA, pH 7.25 (capacitating MW) [31]. Spermatozoa were incubated in $500 \mu \mathrm{L}$ aliquots in polyvinyl alcohol-coated $5 \mathrm{~mL}$ round-bottom tubes at $37^{\circ} \mathrm{C}$ in a humidified atmosphere for 6 hours. The motility was visually evaluated under a microscope (Olympus, IMT-2, Paris, France) at the beginning and at the end of the incubation period. Spermatozoa were then diluted at $5 \times 10^{6} /$ $\mathrm{mL}$ in capacitating MW supplemented with $5 \mathrm{mM}$ procaine to induce hyperactivated motility. Droplets of $100 \mu \mathrm{L}$ of spermatozoa suspension were laid down onto culture dishes and covered with mineral oil.

After preincubation with oviductal fluid, horse and donkey oocytes were washed in capacitating MW and groups of 10 were transferred to droplets of $100 \mu \mathrm{L}$ of donkey spermatozoa suspension in capacitating MW supplemented with $5 \mathrm{mM}$ procaine. Oocytes were co-incubated for 18 hours in an atmosphere of $5 \% \mathrm{CO}_{2}$ in air at $38.5^{\circ} \mathrm{C}$ in $100 \%$ humidity.
For the control group without spermatozoa, horse and donkey oocytes were transferred to droplets of $100 \mu \mathrm{L}$ of capacitating MW supplemented with $5 \mathrm{mM}$ procaine without donkey spermatozoa. They were incubated for 18 hours in an atmosphere of $5 \% \mathrm{CO}_{2}$ in air at $38.5^{\circ} \mathrm{C}$ in $100 \%$ humidity.

\subsubsection{In Vitro Culture of Zygotes}

After 18 hours incubation with or without spermatozoa, zygotes were washed and flushed to remove attached spermatozoa in Dulbecco Modified Eagle Medium (DMEM)-F12 with L-glutamine and HEPES (ref D8900) supplemented with $1.2 \mathrm{~g} / \mathrm{L} \mathrm{NaHCO}_{3}, 10 \%$ FCS, and $25 \mu \mathrm{g} / \mathrm{mL}$ gentamycin (culture medium). Groups of 10 were transferred to droplets of $30 \mu \mathrm{L}$ of culture medium for 30 hours (48 hours post-IVF) in an atmosphere of $5 \% \mathrm{CO}_{2}, 5 \% \mathrm{O}_{2}$, and $90 \% \mathrm{~N}_{2}$ at $38.5^{\circ} \mathrm{C}$ in $100 \%$ humidity.

\subsection{Assessment of Nuclear Status}

Nuclear status was assessed either after $0,24,30,34$, or 38 hours IVM or 48 hours after IVF. Oocytes and zygotes were washed by aspiration in and out of a pipette in PBS, fixed in $4 \%$ paraformaldehyde in PBS for 20 minutes at room temperature, washed in PBS, and processed for analysis.

After IVM, only DNA staining was performed. Oocytes were incubated with $1 \mu \mathrm{g} / \mathrm{mL}$ bisbenzimide (Hoechst 33342) in PBS for 5 minutes and mounted on microscope slides in Mowiol V4-88 (133 mg/mL; Hoechst, Frankfurt, Germany) and n-propyl gallate (5 mg/mL).

DNA and nuclear membrane staining were performed on zygotes 48 hours after IVF. They were incubated for 30 minutes at room temperature in $0.2 \%$ Triton X-100 in PBS. Nonspecific reactions were blocked by incubation for 1 hour at room temperature in $10 \%$ goat serum in PBS. Oocytes and zygotes were incubated overnight at $4^{\circ} \mathrm{C}$ or 2 hours at room temperature with an anti-lamin $\mathrm{A} / \mathrm{C}$ antibody (Thermo scientific) diluted 1:100 in PBS containing $0.2 \%$ BSA and $0.1 \%$ Tween. After four washes for 5 minutes in PBS containing $0.2 \%$ BSA and $0.1 \%$ Tween, they were incubated for 1 hour at room temperature with an Alexa Fluor 594-conjugated anti-mouse antibody diluted 1:400 in PBS. They were then washed five times for 5 minutes in PBS containing $0.1 \%$ Tween and 2 times for 5 minutes in PBS. They were incubated with $1 \mu \mathrm{g} / \mathrm{mL}$ bisbenzimide (Hoechst 33342) in PBS for 5 minutes and mounted on microscope slides in Mowiol V4-88 (133 mg/mL; Hoechst, Frankfurt, Germany) and n-propyl gallate $(5 \mathrm{mg} / \mathrm{mL})$. The slides were kept at $4{ }^{\circ} \mathrm{C}$ in darkness until observation under an epifluorescence microscope (Zeiss). Controls were performed using no primary antibodies to ascertain the absence of nonspecific binding or no secondary antibodies to ascertain the absence of autofluorescence.

\subsection{Statistical Analysis}

The percentages of mature oocytes were compared using chisquare analysis. The percentages of oocytes with two pronuclei were compared using Yates' chi-square analysis. Differences were considered statistically significant at $P<.05$.

\section{Results}

\subsection{Oocyte Recovery}

\subsubsection{Equine Oocytes}

For equine oocytes collection, a total of 28 ovaries were recovered in a local slaughterhouse, and 67 oocytes were collected (2.4 oocyte/ovary). 


\subsubsection{Donkey Oocytes}

Year 1: A total of 22 OPU were performed on adult cyclic jennies during follicular phase during five puncture session. From the five puncture sessions, 193 follicles were flushed, and 92 COCs were collected (48\%), with an increase of the recovery rate along the puncture sessions from $34 \%$ to $56 \%$. Globally, we collected an average of 4.2 COCs per female (Table 1 ).

These oocytes were used for the IVF study in this report.

Year 2: A total of 20 OPU were performed on adult cyclic jennies during follicular phase during puncture session. From the six puncture sessions, 198 follicles were flushed, and 96 COCs were collected (48\%) Globally, we collected an average of 4.8 COCs per female.

These oocytes were used for other studies not included in this report.

Year 3: A total of 10 OPU were performed on adult cyclic jennies during follicular phase during four puncture session. From the four puncture sessions, 110 follicles were flushed, and 63 COCs were collected (57\%) Globally, we collected an average of 6.3 COCs per female.

These oocytes were used for other studies not included in this report.

During the three consecutive breeding seasons, from 2015 to 2017, we performed a total of 52 OPU's, during which 251 oocytes were retrieved from 501 aspirated follicles (collection rate 51\%). This represents an average of 5.1 oocytes collected per jenny during an OPU procedure.

\subsection{Cumulus Aspect After IVM}

At collection, four donkey COCs were expanded, and 88 were compact (96\%; Fig. 1A). All donkey COCs were expanded after over 24 hours IVM (Fig. 1B) [21].

At collection, four equine COCs were expanded, and 63 were compact (94\%). All equine COCs were expanded after over 24 hours IVM.

\subsection{Nuclear Stage of Donkey $(n=49)$ and Equine $(n=12)$ Oocytes After IVM}

At collection ( 0 hours post-IVM), 14 jennies oocytes were analyzed. Most of them contained a germinal vesicle, either with distinct chromatin filaments (6/14; filamentous germinal vesicle;
Fig. 1C) or with chromatin partly condensed (7/14; partly condensed germinal vesicle; Fig. 1D). Nine jennies oocytes were analyzed after 24 hours IVM. Most of them contained a germinal vesicle, either with distinct chromatin filaments (4/9; Fig. 1C) or with chromatin partly condensed (2/9; Fig. 1D), and two of them contained condensed chromatin (Fig. 1E). Nine jennies oocytes were analyzed after 30 hours IVM. Four of them contained a germinal vesicle with distinct chromatin filaments (Fig. 1C), and four of them contained a metaphase 1 (Fig. 1F). Nine jennies oocytes were analyzed after 34 hours IVM. Four of them contained a metaphase 2 with a polar body (Fig. 1G). Eight oocytes were analyzed after 38 hours IVM. Two of them contained a metaphase 2 starting to decondense (Fig. $1 \mathrm{H}$ ), two contained condensed chromatin, and two contained partly condensed germinal vesicle [21].

Equine oocytes were analyzed after 24 hours $(n=6)$ or 30 hours $(n=6)$ IVM. After 24 hours IVM, half of the equine oocytes were in metaphase 2. After 30 hours IVM, four out of six of the equine oocytes were in metaphase 2 , the two other being degenerated.

\subsection{Nuclear Stage of Donkey $(n=42)$ and Equine $(n=55)$ Oocytes 48 Hours After IVF}

Oocytes from donkey jennies and oocytes from pony mares were co-incubated with or without donkey sperm and analyzed 48 hours later. They contained condensed chromatin (Fig. 1E), metaphase 2 (Fig. 1G), one pronucleus (Figs. 1I and 1J) or two pronuclei (Figs. $1 \mathrm{~K}$ and $1 \mathrm{~L}$ ). The percentage of mature oocytes (metaphase II +1 pronucleus +2 pronuclei) was not significantly different between donkey $(34 / 42,81 \%)$ and equine $(37 / 55,67 \%)$ oocytes (chi-squared test, $P>.05$ ). The percentage of oocytes containing two pronuclei after incubation with donkey sperm was not significantly different between donkey (15\%) and equine (21\%) (Yates' chi-squared test, $P>.05$ ). The percentage of oocytes containing two pronuclei was not significantly different after incubation with versus without sperm in the donkey (15\% vs. $6 \%$ ) or in the equine ( $21 \%$ vs. $11 \%$ ) (Yates' chi-squared test, $P>.05)$ [21].

\section{Discussion}

Most wild donkeys are currently endangered or threatened. Likewise, many domestic donkey breeds are at risk of extinction due to the loss of their use for labor in many parts of the world.

Table 1

Oocyte recovery rate from jennies by ovum pick up.

\begin{tabular}{|c|c|c|c|c|c|}
\hline Year & $\begin{array}{l}\text { Number of OPU } \\
\text { per Session }\end{array}$ & $\begin{array}{l}\text { Number of } \\
\text { Follicles Punctured }\end{array}$ & Number of Oocytes Recovered & Recovery Rate per Follicle (\%) & Number of Oocyte per Jenny \\
\hline \multirow[t]{5}{*}{ Year 1} & 4 & 32 & 11 & 34 & 2.75 \\
\hline & 5 & 37 & 16 & 43 & 3.2 \\
\hline & 4 & 31 & 16 & 52 & 4.0 \\
\hline & 3 & 38 & 18 & 47 & 6.0 \\
\hline & 6 & 55 & 31 & 56 & 5.2 \\
\hline Total year 1 & 22 & 193 & 92 & 48 & 4.2 \\
\hline \multirow[t]{6}{*}{ Year 2} & 5 & 41 & 13 & 32 & 2.6 \\
\hline & 4 & 33 & 17 & 52 & 4.3 \\
\hline & 3 & 28 & 9 & 32 & 3.0 \\
\hline & 2 & 43 & 23 & 53 & 11.5 \\
\hline & 3 & 30 & 19 & 63 & 6.3 \\
\hline & 3 & 23 & 15 & 65 & 5.0 \\
\hline Total year 2 & 20 & 198 & 96 & 48 & 4.8 \\
\hline \multirow[t]{4}{*}{ Year 3} & 2 & 24 & 17 & 71 & 8.5 \\
\hline & 2 & 30 & 20 & 67 & 10.0 \\
\hline & 3 & 19 & 11 & 58 & 3.7 \\
\hline & 3 & 37 & 15 & 41 & 5.0 \\
\hline Total year 3 & 10 & 110 & 63 & 57 & 6.3 \\
\hline Total & 52 & 501 & 251 & 51 & 5.1 \\
\hline
\end{tabular}

Abbreviation: OPU, ovum pick up. 


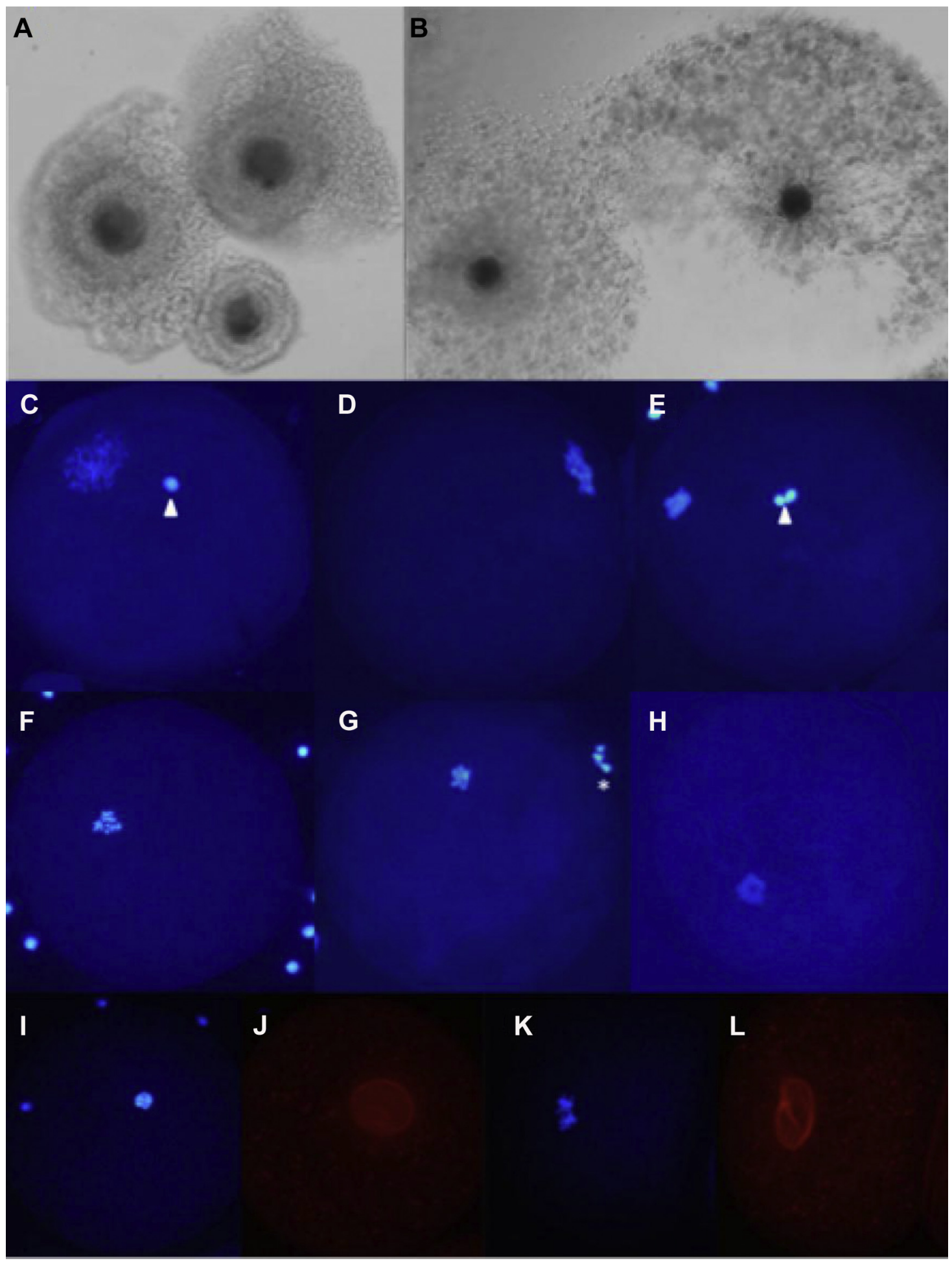

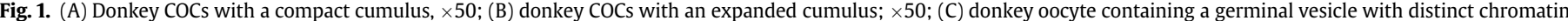

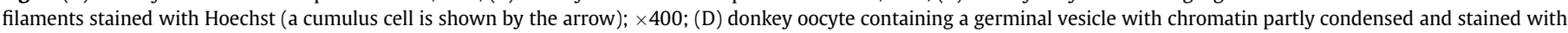

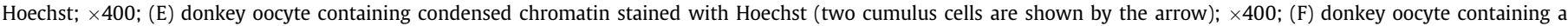

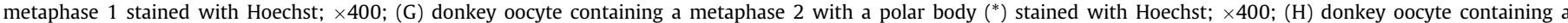

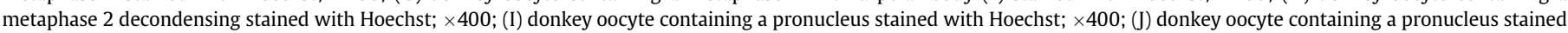

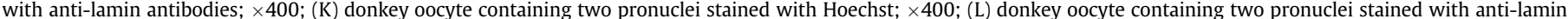
antibodies; $\times 400$. 
Embryo cryopreservation allows the preservation of genetics from both male and female and is the fastest method to restore a breed [6]. The size of the embryos collected in vivo makes them poor candidates for cryopreservation as smaller embryos have been shown to be more resistant to the deleterious effects of cryopreservation in horses [10]. In vitro production of small size donkey embryos appears as a key point for the preservation of endangered donkey breeds. To date, no conventional IVF technique is available in the equine [24-30]. Consequently, intracytoplasmic sperm injection (ICSI) has been widely adopted to produce horse embryos in vitro, both for scientific purposes and in the horse breeding industry $[10,37]$. However, ICSI remains a demanding technique requiring specific and expensive equipment and a strong expertise in micromanipulation. Scarce availability of equids oocytes is a likely explanation for the slow progress of IVP by comparison with the bovine for example. Most oocytes used for research in the equine are obtained by OPU. Transvaginal ultrasound-guided follicular puncture was developed in the mare [38,39]. And OPU has been since extensively used in the equine [10-20]. The mean number of follicles punctured per mare per session ranges from 2 to 12 with an effect of mare [18], breed (lower number in pony mares compared with saddle mares) [11], the estrous cycle stage [13,14], and season [16]. We recently described the establishment of the OPU technique in jennies [21]. In the present report, the mean number of immature oocytes recovered from jennies over a 3 season period was 5.1 (range 2.75-11). The mean oocyte recovery rate per immature follicle over the same period was $51 \%$ (range $34 \%-71 \%$ ). These results are very similar to data reported in the mare, as the mean number of immature oocytes recovered per mare per aspiration session ranges from 0 to 5 , and the oocyte recovery rate per immature follicle in the mare ranges from $20 \%$ to $56 \%$ [11-14,16-18]. All operators were highly experienced in OPU in the mare. Although not statistical, the rapid increase in number of retrieved oocyte per punctured follicle from one session to the other suggests that the operators' skills in OPU in mares can reasonably quickly be transferred to jennies. During the last season, because the procedure was considered safe and to prevent development of resistance to antibiotics, their routine administration at the end of the OPU has been discontinued. No complication following the interruption of systematic antibiotic administration has been observed. To date, only two reports about IVM of abattoirderived donkey oocytes have been published to the best of our knowledge [22,23]. In 2011, Zhao et al used TCM199 or DMEM-F12 supplemented with FCS, porcine follicle stimulating hormone, equine luteinizing hormone, insulin-like growth factor, insulintransferrin-selenium, taurine, L-cysteine, L-glutamine, sodium pyruvate, and gentamycin. The percentage of metaphase 2 was 55\% for compact COCs cultured for 30-36 hours and 77\% for expanded COCs cultured for 24-30 hours, with no significant differences between media. In 2014, Abdoon et al [23] used DMEM with high glucose, DMEM with low glucose, DMEM-F12, TCM199, TCM199F12, or CR1aa medium supplemented with FCS, follicle stimulating hormone, hCG, and gentamicin, the percentage of metaphase 2 after 36 hours of culture was $41 \%, 39 \%, 46 \%, 56 \%, 69 \%$, and $62 \%$, respectively [23]. This demonstrates that TCM199 seems to be an efficient basal medium for jennies oocyte maturation. In our study, IVM of jennies oocytes was performed in TCM199 supplemented with FCS and EGF. This medium has been used in the equine with high maturation rates (64\% [33], 65-74\% [40], 73-76\% [41], 71\% [17], 61\% [35], and 67\% in this study). In the present work, this medium was efficient to sustain cumulus expansion in donkey oocytes because all donkey COCs were expanded after over 24 hours IVM. Though Zhao et al [22] used 30-36 hours and 24-30 hours for compact and expanded COCs, respectively, the optimal duration of in vitro culture has never been evaluated in donkey oocytes. Therefore, we analyzed the chronology of IVM from 0 to 38 hours in culture. At collection, jennies oocytes contained a germinal vesicle, either with distinct chromatin filaments or with chromatin partly condensed. These meiotic stages were observed in equine oocytes immediately after in vivo collection $[42,43]$. After 24 hours IVM, some jennies oocytes contained condensed chromatin, and metaphase 1 oocytes were observed after 30 hours. In the equine oocytes, metaphase 1 was observed after 24 hours IVM which agrees with previous articles reporting that $25 \%-37 \%$ of the equine oocytes are in metaphase 1 after 24 hours of IVM $[43,44]$. In our conditions, equine oocytes seem to reach the metaphase 1 stage faster than donkey oocytes. About half of the donkey oocytes were in metaphase 2 after 34 hours IVM in our conditions, whereas half of equine oocytes were in metaphase 2 after only 24 hours, which is consistent with previous articles reporting that $25 \%-35 \%$ of the equine oocytes are in metaphase 2 after 24 hours IVM $[43,44]$. In our conditions, IVM of oocytes from jennies is slower than that of equine oocytes. Finally, after 38 hours IVM, $25 \%$ of donkey oocytes contained metaphase 2 starting to decondense, and $25 \%$ were degenerated. Based on our observation, we suggest that the optimal duration for IVM of donkey oocytes should be 34 hours. Our data are consistent with the results from Zhao et al [22], who obtained 55\% of metaphase 2 oocytes from jennies after 30-36 hours of IVM. Abdoon et al [23] obtained higher IVM rates with donkey oocytes after 36 hours of culture using TCM199-F12 or CR1aa medium supplemented with FCS, follicle stimulating hormone, hCG, and gentamicin (69\% and 62\%). Thus, our IVM medium, though efficient for equine oocytes, may not be optimal for donkey oocytes.

Post-IVM jennies oocytes were co-incubated with donkey sperm treated with procaine, as previously described for equine gametes [31]. Equine oocytes were used as controls. A 60\% rate of equine IVF was reported after treatment of equine spermatozoa with procaine $[31,35]$. However, in our study, only $15 \%$ of donkey oocytes and $21 \%$ of equine oocytes contained two pronuclei after co-incubation with donkey spermatozoa treated with procaine. None of them developed further after 48 hours post-IVF, suggesting that treatment with procaine is not efficient for IVF. Moreover, when donkey or equine oocytes were co-incubated without spermatozoa, $6 \%$ and $11 \%$, respectively, contained two pronuclei. Procaine has been shown to induce cytokinesis in equine oocytes [32]. Our study has demonstrated that, like in the horse, treatment procaine induces cytokinesis in jennies oocytes. The procaïneinduced cytokinesis in equine oocytes is not stimulated by a rise in cytoplasmic calcium in contrast to the situation in which oocytes are fertilized by ICSI or parthenogenetically activated by ionomycin [32]. Success of IVF based on the presence of two pronuclei at 20-24 hours after sperm-oocyte co-incubation without signs of spermatozoal penetration and/or observation of a second polar body should be cautiously evaluated.

\section{Conclusion}

We recently established for the first time conditions for ovum pick in jennies [21]. After three seasons, it can be considered that collection rates similar or even slightly better than those reported in the equine have been rapidly reached. While OPU in jennies is perceived by the staff as physically more demanding, yields seem to be improving as more experience is gained. It has been shown that IVM of donkey oocytes can produce $44 \%$ of metaphase 2 in our conditions. More studies on conditions for IVF and development of donkey zygotes are in progress. Our study contributes to the development of in vitro techniques for donkey embryo production. This is of major importance for preservation of genetics from wild and domestic endangered donkey breeds. 


\section{Acknowledgments}

The authors wish to thank the staff of the experimental stud at the "Unité Expérimentale de Physiologie Animale de l'Orfrasière (UEPAO)" for technical help. This work has benefited from the facilities and expertise of the "Plateforme d'Imagerie Cellulaire" (PIC) of the UMR 85 "Physiologie de la Reproduction et des Comportements."

Carla Moros-Nicolás has a postdoctoral fellowship from Fundación Séneca, Agencia de Ciencia yTecnología de la Región de Murcia, Spain.

Financial disclosure.

This work was financially supported by the "Institut Français du Cheval et de l'Equitation" (IFCE) and the French National Infrastructure of Research CRB anim funded by "Investissements d'avenir", ANR-11-INBS-0003.

\section{References}

[1] Quaresma M, Martins AM, Rodrigues JB, Colaco J, Payan-Carreira R. Pedigree and herd characterization of a donkey breed vulnerable to extinction. Animal 2014;8:354-9.

[2] Rizzi R, Tullo E, Cito AM, Caroli A, Pieragostini E. Monitoring of genetic diversity in the endangered Martina Franca donkey population. J Anim Sci 2011;89:1304-11.

[3] Tosi U, Bernabo N, Verni F, Valbonetti L, Muttini A, Mattioli M, Barboni B. Postpartum reproductive activities and gestation length in Martina Franca jennies, an endangered Italian donkey breed. Theriogenology 2013;80:120-4.

[4] Cortes-Gutierrez EI, Crespo F, Gosalvez A, Davila-Rodriguez MI, Lopez Fernandez C, Gosalvez J. DNA fragmentation in frozen sperm of Equus asinus: Zamorano-Leones, a breed at risk of extinction. Theriogenology 2008;69: 1022-32.

[5] Smits K, Hoogewijs M, Woelders H, Daels P, Van Soom A. Breeding or assisted reproduction? Relevance of the horse model applied to the conservation of endangered equids. Reprod Domest Anim 2012;47 Suppl 4:239-48.

[6] Gandini G, Pizzi F, Stella A, Boettcher PJ. The costs of breed reconstruction from cryopreserved material in mammalian livestock species. Genet Sel Evol 2007;39:465-79.

[7] Camillo F, Panzani D, Scollo C, Rota A, Crisci A, Vannozzi I, Balbo S. Embryo recovery rate and recipients' pregnancy rate after nonsurgical embryo transfer in donkeys. Theriogenology 2010;73:959-65.

[8] Panzani D, Rota A, Crisci A, Kindahl H, Govoni N, Camillo F. Embryo quality and transcervical technique are not the limiting factors in donkey embryo transfer outcome. Theriogenology 2012;77:563-9.

[9] Meyers-Brown G, Bidstrup LA, Famula TR, Colgin M, Roser JF. Treatment with recombinant equine follicle stimulating hormone (reFSH) followed by re combinant equine luteinizing hormone (reLH) increases embryo recovery in superovulated mares. Anim Reprod Sci 2011;128:52-9.

[10] Hinrichs K. Assisted reproduction techniques in the horse. Reprod Fertil Dev 2012;25:80-93.

[11] Duchamp G, Bézard J, Palmer E. Oocyte yield and the consequences of puncture of all follicles larger than 8 millimeters in mares. Biol Reprod Monogr Ser 1995;1:233-41.

[12] Kanitz W, Becker F, Alm H, Torner H. Ultrasound-guided follicular aspiration in mares. Biol Reprod Monogr Ser 1995;1:225-31.

[13] Goudet G, Bézard J, Duchamp G, Gérard N, Palmer E. Equine oocyte competence for nuclear and cytoplasmic in vitro maturation: effect of follicle size and hormonal environment. Biol Reprod 1997:57:232-45.

[14] Goudet G, Bézard J, Belin F, Duchamp G, Palmer E, Gérard N. Oocyte competence for in vitro maturation is associated with histone H1 kinase activity and is influenced by estrous cycle stage in the mare. Biol Reprod 1998;59:456-62.

[15] Marchal R, Caillaud M, Martoriati A, Gerard N, Mermillod P, Goudet G. Effect of growth hormone $(\mathrm{GH})$ on in vitro nuclear and cytoplasmic oocyte maturation, cumulus expansion, hyaluronan synthases, and connexins 32 and 43 expression, and $\mathrm{GH}$ receptor messenger RNA expression in equine and porcine species. Biol Reprod 2003;69:1013-22.

[16] Purcell SH, Seidel GE, McCue PM, Squires EL. Aspiration of oocytes from transitional, cycling, and pregnant mares. Anim Reprod Sci 2007;100: 291-300.

[17] Deleuze S, Goudet G, Caillaud M, Lahuec C, Duchamp G. Efficiency of embryonic development after intrafollicular and intraoviductal transfer of in vitro and in vivo matured horse oocytes. Theriogenology 2009:72:203-9.

[18] Jacobson CC, Choi YH, Hayden SS, Hinrichs K. Recovery of mare oocytes on a fixed biweekly schedule, and resulting blastocyst formation after intracytoplasmic sperm injection. Theriogenology 2010;73:1116-26.

[19] Franciosi F, Lodde V, Goudet G, Duchamp G, Deleuze S, Douet C, Tessaro I, Luciano AM. Changes in histone $\mathrm{H} 4$ acetylation during in vivo versus in vitro maturation of equine oocytes. Mol Hum Reprod 2012;18:243-52.
[20] Galli C, Duchi R, Colleoni S, Lagutina I, Lazzari G. Ovum pick up, intracytoplasmic sperm injection and somatic cell nuclear transfer in cattle, buffalo and horses: from the research laboratory to clinical practice. Theriogenology 2014;81:138-51.

[21] Goudet G, Douet C, Kaabouba-Escurier A, Couty I, Moros-Nicolas C, Barriere P, Blard T, Reigner F, Deleuze S, Magistrini M. Establishment of conditions for ovum pick up and IVM of jennies oocytes toward the setting up of efficient IVF and in vitro embryos culture procedures in donkey (Equus asinus). Theriogenology 2016:86:528-35.

[22] Zhao G, Wu K, Cui L, Zhao L, Liu Y, Tan X, Zhou H. In vitro maturation and artificial activation of donkey oocytes. Theriogenology 2011;76:700-4.

[23] Abdoon AS, Abdel-Rahman HA, Shawki SM, Kandil OM, Fathalla SI. Influence of follicle size, methods of retrieval on oocytes yield and morphology in Egyptian Jennies ovaries with special reference to maturation rate in vitro. Vet Res Commun 2014;38:287-95.

[24] Bézard J, Magistrini M, Duchamp G, Palmer E. Chronology of equine fertilisation and embryonic development in vivo and in vitro. Equine Vet J Suppl 1989;8:105-10.

[25] Palmer E, Bezard J, Magistrini M, Duchamp G. In vitro fertilization in the horse. A retrospective study. J Reprod Fertil Suppl 1991;44:375-84.

[26] Alm H, Torner H, Blottner S, Nurnberg G, Kanitz W. Effect of sperm cryopreservation and treatment with calcium ionophore or heparin on in vitro fertilization of horse oocytes. Theriogenology 2001;56:817-29.

[27] Hinrichs K, Love CC, Brinsko SP, Choi YH, Varner DD. In vitro fertilization of in vitro-matured equine oocytes: effect of maturation medium, duration of maturation, and sperm calcium ionophore treatment, and comparison with rates of fertilization in vivo after oviductal transfer. Biol Reprod 2002;67: 256-62.

[28] Mugnier S, Kervella M, Douet C, Canepa S, Pascal G, Deleuze S, Duchamp G, Monget P, Goudet G. The secretions of oviduct epithelial cells increase the equine in vitro fertilization rate: are osteopontin, atrial natriuretic peptide A and oviductin involved? Reprod Biol Endocrinol 2009;7:129.

[29] Dell'aquila ME, Fusco S, Lacalandra GM, Maritato F. In vitro maturation and fertilization of equine oocytes recovered during the breeding season. Theriogenology 1996;45:547-60.

[30] Dell'Aquila ME, Cho YS, Minoia P, Traina V, Lacalandra GM, Maritato F. Effects of follicular fluid supplementation of in-vitro maturation medium on the fertilization and development of equine oocytes after in-vitro fertilization or intracytoplasmic sperm injection. Hum Reprod 1997;12:2766-72.

[31] McPartlin LA, Suarez SS, Czaya CA, Hinrichs K, Bedford-Guaus SJ. Hyperactivation of stallion sperm is required for successful in vitro fertilization of equine oocytes. Biol Reprod 2009;81:199-206.

[32] Leemans B, Gadella BM, Stout TA, Heras S, Smits K, Ferrer-Buitrago M, Claes E, Heindryckx B, De Vos WH, Nelis H, Hoogewijs M, Van Soom A. Procaine induces cytokinesis in horse oocytes via a pH-Dependent mechanism. Biol Reprod 2015;93:23.

[33] Goudet G, Belin F, Mlodawska W, Bezard J. Influence of epidermal growth factor on in vitro maturation of equine oocytes. J Reprod Fertil Suppl 56, 2000: 483-92.

[34] Pillet E, Duchamp G, Batellier F, Beaumal V, Anton M, Desherces S, Schmitt E, Magistrini M. Egg yolk plasma can replace egg yolk in stallion freezing extenders. Theriogenology 2011;75:105-14.

[35] Ambruosi B, Accogli G, Douet C, Canepa S, Pascal G, Monget P, Moros C, Holmskov U, Mollenhauer J, Robbe-Masselot C, Vidal O, Desantis S, Goudet G. Deleted in malignant brain tumor 1 is secreted in the oviduct and involved in the mechanism of fertilization in equine and porcine species. Reproduction 2013;146:119-33.

[36] Carrasco LC, Romar R, Aviles M, Gadea J, Coy P. Determination of glycosidase activity in porcine oviductal fluid at the different phases of the estrous cycle. Reproduction 2008;136:833-42.

[37] Choi YH, Varner DD, Love CC, Hartman DL, Hinrichs K. Production of live foals via intracytoplasmic injection of lyophilized sperm and sperm extract in the horse. Reproduction 2011;142:529-38.

[38] Bruck I, Raun K, Synnestvedt B, Greve T. Follicle aspiration in the mare using a transvaginal ultrasound-guided technique. Equine Vet J 1992:24:58-9.

[39] Cook NL, Squires EL, Ray BS, Cook VM, Jasko DJ. Transvaginal ultrasonically guided follicular aspiration of equine oocytes - preliminary results. J Equine Vet Sci 1992;12:204-7.

[40] Dell'Aquila ME, Caillaud M, Maritato F, Martoriati A, Gérard N, Aiudi G, Minoia P, Goudet G. Cumulus expansion, nuclear maturation and connexin 43, cyclooxygenase- 2 and FSH receptor mRNA expression in equine cumulusoocyte complexes cultured in vitro in the presence of FSH and precursors for hyaluronic acid synthesis. Reprod Biol Endocrinol 2004;2:44.

[41] Luciano AM, Goudet G, Perazzoli F, Lahuec C, Gérard N. Glutathione content and glutathione peroxidase expression in in vivo and in vitro matured equine oocytes. Mol Reprod Dev 2006;73:658-66.

[42] Bezard J, Bogh IB, Duchamp G, Hyttel P, Greve T. Comparative evaluation of nuclear morphology of equine oocytes aspirated in vivo and stained with Hoechst and orcein. Cells Tissues Organs 2002;170:228-36.

[43] Hinrichs K, Schmidt AL, Friedman PP, Selgrath JP, Martin MG. In vitro maturation of horse oocytes: characterization of chromatin configuration using fluorescence microscopy. Biol Reprod 1993;48:363-70.

[44] Tremoleda JL, Schoevers EJ, Stout TA, Colenbrander B, Bevers MM. Organisation of the cytoskeleton during in vitro maturation of horse oocytes. Mol Reprod Dev 2001;60:260-9. 